\title{
Liver
}

\section{PTH-071 DECOMPENSATED ALCOHOLIC LIVER DISEASE AND BIRTH WEIGHT}

doi:10.1136/gut.2011.239301.472

A Ali, ${ }^{1}{ }^{*} \mathrm{~J}$ Jones, ${ }^{1}$ M P Bradley, ${ }^{1}$ R Davies, ${ }^{1}$ R J Beck, ${ }^{2}$ D Gleeson' ${ }^{1}$ Liver Unit, Sheffield Teaching Hospitals, Sheffield, UK; ${ }^{2}$ Department of Radiology, Sheffield Teaching Hospitals, Sheffield, UK

Introduction Only a minority of heavy drinkers develop serious alcoholic liver disease (ALD). Low birth weight has been associated with a several adult-onset diseases such as ischemic heart disease, hypertension and type 2 diabetes. ${ }^{1}$ Its association with ALD is not established. Using case-control strategy the authors aim to assess the associations between decompensated ALD and birth weight, prematurity and maternal smoking during pregnancy.

Methods The authors recruited two cohorts of heavy drinkers ( $\geq 60 \mathrm{U} /$ week (men) and $\geq 40 \mathrm{U} /$ week (women) for $\geq 5$ years). Cases ( $n=215,136$ men, age (mean \pm SD) $46 \pm 9$ years) had decompensated ALD (Child's grade B or C). Controls ( $n=163$,
126 men, age $47 \pm 10$ years) had no clinical evidence of liver disease, persistently normal serum bilirubin, albumin and prothrombin time and ultrasound showing normal or echobright texture and no other abnormality. Data were collected regarding subjects' birth weight, gestational age (GA) at birth ( $\mathrm{n}=200$ for cases, 148 for controls), and mother's smoking status during pregnancy (recalled by subjects, following discussion with mother when possible) ( $\mathrm{n}=148$ cases, 109 controls). Birth weight was classified as very low ( $<1.5 \mathrm{~kg})$, low $(1.5-2.5$ $\mathrm{kg}$ ), normal $(2.5-4 \mathrm{~kg})$ and high $(>4 \mathrm{~kg})$. Comparison between groups was by Student $t$ and $\chi^{2}$ tests. Recalled birth weights were also compared with actual birth weights in Sheffield hospital records, when these were available (22 cases, 13 controls) and close correlations were observed $(r=0.80$ for cases and 0.76 for controls; $\mathrm{p}<0.001$ and 0.002 ).

Results See table 1.

There were no differences in birth weight or prevalence of low birth weight between males and females or between cases and controls when analysis was confined to males or to females.

Conclusion In this case-control study, the authors found no association between presence of ALD in heavy drinkers and either low birth weight or prematurity.

Competing interests None.

Keywords alcoholic liver disease, birth weight.

\section{REFERENCE}

1. Phillips DIW. Birth weight and adulthood disease and the controversies. Fetal Matern Med Rev 2006;17:205-227.

Table 1 PTH-071

\begin{tabular}{llll}
\hline & Cases & p Value & Controls \\
\hline Premature delivery (gestational age $<37$ weeks) (\%) & $17(8.5)$ & NS & $11(7.4)$ \\
Very low/low/normal/high birthweight distribution. & $6 / 25 / 159 / 25$ & NS & $4 / 14 / 130 / 14$ \\
Birth weight $(\mathrm{kg})$ & $3.2 \pm 0.74$ & NS & $3.2 \pm 0.75$ \\
Birth weight $(\mathrm{kg})$ in full term babies & $3.3 \pm 0.64$ & NS & $3.4 \pm 0.61$ \\
Birth weight/gestational age ratio (g/week) & $81 \pm 17$ & NS & $83 \pm 17$ \\
Mother smoking during pregnancy (\%) & $72(48.6)$ & NS & $48(44.0)$ \\
\hline
\end{tabular}

